\title{
Two Methods of Solving Heat and Wave Problems
}

R.T. COUTO ${ }^{1}$, Depto. Matemática Aplicada, Universidade Federal Fluminense (UFF), Campus do Valonguinho, Centro, 24020-120 Niterói, RJ, Brazil.

\begin{abstract}
Two methods of solving a large class of heat and wave problems are described. The problems are two-dimensional and exhibit not only non-homogeneity of the differential equation and of the initial and boundary conditions but also time dependence of the sources and of the initial and boundary data. The two corresponding solutions are proved to be equal, and an illustration of the methods is provided by applying them to solve a specific problem using the polar coordinates.
\end{abstract}

\section{Introduction}

In this work, we solve a large class of two-dimensional heat and wave problems by means of two different methods. They are powerful methods which are capable of dealing with a variety of troublesome problems analytically, in a compact and uniform way. In fact, the heat and wave problems solved here present non-homogeneity of the differential equation and of the initial and boundary conditions as well as time dependence of the sources and of the initial and boundary data.

We develop such methods by generalizing those described in Reference $[2$, Sec. 9.17]. The generalization achieved allows the application of the methods to problems with more than one spatial dimension and under boundary conditions of the first (Dirichlet), second (Neumann) or third types. We also confirm that the solutions derived with the two methods are equal. In addition, we illustrate the methods by applying them to solve a specific heat problem.

The fact that we have considered two spatial dimensions to develop the methods offers no difficult in applying them to three-dimensional problems. Moreover, since the main features of the methods do not depend on the kind of the problem (heat or wave), we expound them by solving the heat problem only. Afterwards, by changing the heat problem solution in an obvious way, we provide the wave problem solution.

In Section 2, we define the heat and wave problems to be solved as well as, for its necessity in the succeeding sections, present the solution for the particular case of homogeneous differential equation and boundary condition. In Sections 3 and 4, we solve the heat problem according to the first and second methods. In Section 5, we exemplify the methods by applying them to solve the Dirichlet problem for the heat equation in a unit disk. In Section 6 , we demonstrate that the solutions obtained with the two methods are equivalent. A section of final comments concludes the body of the paper.

\footnotetext{
${ }^{1}$ toscano@im.uff.br
} 


\section{Formulation of the Problem}

Consider a bounded domain $\mathcal{A} \subset \mathbb{R}^{2}$, and let us denote its boundary by $\partial \mathcal{A}$ and the closure $\mathcal{A} \cup \partial \mathcal{A}$ by $\overline{\mathcal{A}}$. We will use the vector variables $\vec{\rho}$ and $\vec{\rho}_{B}$ to refer to a generic point of $\overline{\mathcal{A}}$ and $\partial \mathcal{A}$, respectively, and this is to be understood unless another use is made.

In this work, we solve the following two initial-boundary value problems: the heat problem

$$
T_{t}(\vec{\rho}, t)-\nabla^{2} T=f(\vec{\rho}, t), \quad \hat{\mathrm{B}} T\left(\vec{\rho}_{B}, t\right)=g\left(\vec{\rho}_{B}, t\right), \quad T\left(\vec{\rho}, t_{0}\right)=h(\vec{\rho})
$$

and the wave problem

$z_{t t}(\vec{\rho}, t)-\nabla^{2} z=f(\vec{\rho}, t), \quad \hat{\mathrm{B}} z\left(\vec{\rho}_{B}, t\right)=g\left(\vec{\rho}_{B}, t\right), \quad z\left(\vec{\rho}, t_{0}\right)=h(\vec{\rho}), \quad z_{t}\left(\vec{\rho}, t_{0}\right)=v(\vec{\rho})$,

with $t \geq t_{0}$. Here, $\hat{\mathrm{B}}$ is the operator

$$
\hat{\mathrm{B}} \equiv p\left(\vec{\rho}_{B}\right)+q\left(\vec{\rho}_{B}\right) \partial / \partial n,
$$

where $\partial / \partial n=\vec{n} \cdot \nabla$, the normal derivative, with the unit normal vector $\vec{n}$ directed outward from $\overline{\mathcal{A}}$ at a point of $\partial \mathcal{A}$. In particular, if $\hat{\mathrm{B}}=1$ or $\hat{\mathrm{B}}=\partial / \partial n$, we have the Dirichlet or the Neumann condition, respectively.

It is well known (c.f. References [2, Ch. 9] and [6, Ch. IX, Sec. 4]) that, when $f=g \equiv 0$, we can use the method of separation of variables, obtaining, for the heat problem, the solution

$$
T(\vec{\rho}, t)=\sum_{\lambda} A_{\lambda} e^{-\lambda t} \psi_{\lambda}(\vec{\rho}),
$$

where the $\lambda$ 's are the eigenvalues and the $\psi$ 's are the eigenfunctions of the eigenvalue problem (studied, for example, in Reference [6, Ch. VIII, Sec. 10], wherein the statements below are also discussed)

$$
\nabla^{2} \psi+\lambda \psi(\vec{\rho})=0, \quad \hat{\mathrm{B}} \psi\left(\vec{\rho}_{B}\right)=0 .
$$

The self-adjointness of $\nabla^{2}$ implies that the eigenvalues are real and justifies the imposition of the orthornormality condition

$$
\int_{\mathcal{A}} d A \psi_{\lambda^{\prime}}^{*}(\vec{\rho}) \psi_{\lambda}(\vec{\rho})=\delta_{\lambda^{\prime} \lambda}
$$

By using it, we can calculate the coefficients of the generalized Fourier series of a function $F(\vec{\rho})$ :

$$
F(\vec{\rho})=\sum_{\lambda} F_{\lambda} \psi_{\lambda}(\vec{\rho}) \quad \Rightarrow \quad F_{\lambda}=\int_{\mathcal{A}} d A \psi_{\lambda}^{*}(\vec{\rho}) F(\vec{\rho}) .
$$

By demanding that (2.4) satisfy the initial condition in (2.1) and using (2.7), we can find $A_{\lambda}$. As a result, we have that the solution of the problem (2.1) without sources $(f \equiv 0)$ and with homogeneous boundary condition $(g \equiv 0)$ is given by

$$
T(\vec{\rho}, t)=\sum_{\lambda} h_{\lambda} e^{-\lambda\left(t-t_{0}\right)} \psi_{\lambda}(\vec{\rho}), \quad \text { with } h_{\lambda}=\int_{\mathcal{A}} d A \psi_{\lambda}^{*}(\vec{\rho}) h(\vec{\rho}) .
$$


In an analogous manner, we obtain the following solution for the wave problem (2.2) when $f=g \equiv 0$ (Reference [6, Ch. VIII, Secs. 8 and 9]):

$$
z(\vec{\rho}, t)=\sum_{\lambda} \psi_{\lambda}(\vec{\rho})\left[h_{\lambda} C_{\lambda}\left(t-t_{0}\right)+v_{\lambda} S_{\lambda}\left(t-t_{0}\right)\right]
$$

where $v_{\lambda}=\int_{\mathcal{A}} d A \psi_{\lambda}^{*}(\vec{\rho}) v(\vec{\rho})$,

$C_{\lambda}(t) \equiv\left\{\begin{array}{cc}1 & (\lambda=0) \\ \cos (t \sqrt{\lambda}) & (\lambda>0)\end{array} \quad\right.$ and $\quad S_{\lambda}(t) \equiv\left\{\begin{array}{cc}t & (\lambda=0) \\ (1 / \sqrt{\lambda}) \sin (t \sqrt{\lambda}) & (\lambda>0) .\end{array}\right.$

That the eigenvalues are nonnegative can be deduced from a physical reasoning (such as that in Reference [5, Sec. 8.2]) or analytically.

\section{The First Method of Solution}

Let us try to obtain the solution of the problem (2.1) in the following form:

$$
T(\vec{\rho}, t)=\sum_{\lambda} T_{\lambda}(t) \psi_{\lambda}(\vec{\rho})
$$

Our goal here is to determine $T_{\lambda}(t)$, which, according to $(2.7)$, can be written as

$$
T_{\lambda}(t)=\int_{\mathcal{A}} d A \psi_{\lambda}^{*}(\vec{\rho}) T(\vec{\rho}, t)
$$

If we differentiate it with respect to $t$ and use the heat equation in (2.1) to replace $T_{t}$ by $\nabla^{2} T+f$, we obtain

$$
\dot{T}_{\lambda}(t)=\int_{\mathcal{A}} d A \psi_{\lambda}^{*}(\vec{\rho}) T_{t}(\vec{\rho}, t)=\int_{\mathcal{A}} d A \psi_{\lambda}^{*} \nabla^{2} T+\int_{\mathcal{A}} d A \psi_{\lambda}^{*} f .
$$

We can develop the integral of $\psi_{\lambda}^{*} \nabla^{2} T$ above by using integration by parts, Helmholtz's equation in (2.5) and Green's second identity as follows:

$$
\begin{aligned}
& \int_{\mathcal{A}} d A \psi_{\lambda}^{*} \nabla^{2} T=\int_{\mathcal{A}} d A[T \overbrace{\nabla^{2} \psi_{\lambda}^{*}}^{-\lambda \psi_{\lambda}^{*}} \nabla \cdot\left(\psi_{\lambda}^{*} \nabla T-T \nabla \psi_{\lambda}^{*}\right)]= \\
& -\lambda \int_{\mathcal{A}} d A \psi_{\lambda}^{*} T+\oint_{\partial \mathcal{A}} d s\left[\psi_{\lambda}^{*} \frac{\partial T}{\partial n}-T \frac{\partial \psi_{\lambda}^{*}}{\partial n}\right]=-\lambda T_{\lambda}(t)+B_{\lambda}(t),
\end{aligned}
$$

where, in the last step, we have used (3.2) and the definition

$$
\oint_{\partial \mathcal{A}} d s\left[\psi_{\lambda}^{*}(\vec{\rho}) \frac{\partial T}{\partial n}(\vec{\rho}, t)-\frac{\partial \psi_{\lambda}^{*}}{\partial n}(\vec{\rho}) T(\vec{\rho}, t)\right] \equiv B_{\lambda}(t) .
$$

Therefore, with the substitution of the result in (3.4) into (3.3), this equation becomes

$$
\dot{T}_{\lambda}(t)+\lambda T_{\lambda}(t)=B_{\lambda}(t)+f_{\lambda}(t)
$$


where we have recognized the last term in (3.3) as the coefficient of the generalized Fourier series of $f(\vec{\rho}, t)$ :

$$
f(\vec{\rho}, t)=\sum_{\lambda} f_{\lambda}(t) \psi_{\lambda}(\vec{\rho}), \text { with } f_{\lambda}(t)=\int_{\mathcal{A}} d A \psi_{\lambda}^{*}(\vec{\rho}) f(\vec{\rho}, t) .
$$

The first-order ODE (3.6) is to be solved under the initial condition $T_{\lambda}\left(t_{0}\right)=h_{\lambda}$, which can be deduced by using the problem initial condition in (2.1) as well as (3.1) and (2.8). It is a simple matter to show that the solution is

$$
T_{\lambda}(t)=h_{\lambda} e^{-\lambda\left(t-t_{0}\right)}+\int_{t_{0}}^{t} d t^{\prime} e^{-\lambda\left(t-t^{\prime}\right)}\left[f_{\lambda}\left(t^{\prime}\right)+B_{\lambda}\left(t^{\prime}\right)\right] .
$$

In summary, by substituting (3.8) into (3.1), we can affirm that the solution of the problem defined by $(2.1)$ is given by

$$
T(\vec{\rho}, t)=\sum_{\lambda} \psi_{\lambda}(\vec{\rho}) e^{-\lambda t}\left\{h_{\lambda} e^{\lambda t_{0}}+\int_{t_{0}}^{t} d t^{\prime} e^{\lambda t^{\prime}}\left[f_{\lambda}\left(t^{\prime}\right)+B_{\lambda}\left(t^{\prime}\right)\right]\right\},
$$

with the $h_{\lambda}, f_{\lambda}(t)$ and $B_{\lambda}(t)$ given in (2.8), (3.7) and (3.5), respectively.

For the wave problem defined by $(2.2)$, an analogous procedure would lead to the solution

$$
z(\vec{\rho}, t)=\sum_{\lambda} \psi_{\lambda}(\vec{\rho})\left\{h_{\lambda} C_{\lambda}\left(t-t_{0}\right)+v_{\lambda} S_{\lambda}\left(t-t_{0}\right)+\int_{t_{0}}^{t} d t^{\prime}\left[f_{\lambda}\left(t^{\prime}\right)+B_{\lambda}\left(t^{\prime}\right)\right] S_{\lambda}\left(t-t^{\prime}\right)\right\} .
$$

\section{The Second Method of Solution}

Another method of solving the heat problem given by (2.1) consists of first homogenizing the boundary condition. This is done by subtracting from $T$ a suitable function $U$ also satisfying the boundary condition, that is, by considering the difference $T-U \equiv T^{\star}$, which satisfies the homogenized boundary condition (that with $g \equiv 0)$. We choose $U$ as the solution of the problem

$$
\nabla^{2} U(\vec{\rho} \mid t)=0, \quad \hat{\mathrm{B}} U\left(\vec{\rho}_{B} \mid t\right)=g\left(\vec{\rho}_{B}, t\right),
$$

where $t$ is viewed as a parameter.

Next we determine the correction $T^{\star}$. It is easily seen to be the solution of the following heat problem:

$T_{t}^{\star}(\vec{\rho}, t)-\nabla^{2} T^{\star}=f(\vec{\rho}, t)-U_{t}(\vec{\rho} \mid t), \quad \hat{\mathrm{B}} T^{\star}\left(\vec{\rho}_{B}, t\right)=0, \quad T^{\star}\left(\vec{\rho}, t_{0}\right)=h(\vec{\rho})-U\left(\vec{\rho} \mid t_{0}\right)$.

In fact, notice that

$$
T(\vec{\rho}, t)=T^{\star}(\vec{\rho}, t)+U(\vec{\rho} \mid t)
$$


satisfies the problem (2.1):

$$
\begin{gathered}
T_{t}(\vec{\rho}, t)-\nabla^{2} T=\underbrace{\left(T_{t}^{\star}-\nabla^{2} T^{\star}\right)}_{f-U_{t}}+(U_{t}-\underbrace{\nabla^{2} U}_{0})=f \checkmark \\
\hat{\mathrm{B}} T\left(\vec{\rho}_{B}, t\right)=\underbrace{\hat{\mathrm{B}} T^{\star}}_{0}+\underbrace{\hat{\mathrm{B}} U}_{g}=g \checkmark \\
T\left(\vec{\rho}, t_{0}\right)=\underbrace{T^{\star}\left(\vec{\rho}, t_{0}\right)}_{h(\vec{\rho})-U\left(\vec{\rho} \mid t_{0}\right)}+U\left(\vec{\rho} \mid t_{0}\right)=h(\vec{\rho}) \checkmark
\end{gathered}
$$

Finally, we apply both the superposition and Duhamel's principles (cf. References [6, Prob. 6.5 of Ch. VIII and Prob. 2.9 of Ch. IX] and [4, Ch. 5, 1, c]) to obtain the solution of the problem (4.2):

$$
T^{\star}(\vec{\rho}, t)=I(\vec{\rho}, t)+\int_{t_{0}}^{t} d t^{\prime} Q\left(\vec{\rho}, t \mid t^{\prime}\right),
$$

where $I(\vec{\rho}, t)$, with $t>t_{0}$, is the solution of

$$
I_{t}(\vec{\rho}, t)-\nabla^{2} I=0, \quad \hat{\mathrm{B}} I\left(\vec{\rho}_{B}, t\right)=0, \quad I\left(\vec{\rho}, t_{0}\right)=h(\vec{\rho})-U\left(\vec{\rho} \mid t_{0}\right),
$$

and $Q\left(\vec{\rho}, t \mid t^{\prime}\right)$, with $t>t^{\prime}>t_{0}$, is the solution of

$$
Q_{t}\left(\vec{\rho}, t \mid t^{\prime}\right)-\nabla^{2} Q=0, \quad \hat{\mathrm{B}} Q\left(\vec{\rho}_{B}, t \mid t^{\prime}\right)=0, \quad Q\left(\vec{\rho}, t^{\prime} \mid t^{\prime}\right)=f\left(\vec{\rho}, t^{\prime}\right)-U_{t^{\prime}}\left(\vec{\rho} \mid t^{\prime}\right) .
$$

These are simpler heat problems, without source and with homogeneous boundary conditions, of the same type of that solved at the end of Section 2. Their solutions are thus given by (2.8), but with the initial temperature $h$ replaced by those given in (4.5) and (4.6). [Notice that, as required by Duhamel's principle, the initial time for the second problem is $t=t^{\prime}$ instead of $t=t_{0}$.] Therefore, we can immediately write

$$
\begin{gathered}
I(\vec{\rho}, t)=\sum_{\lambda}\left[h_{\lambda}-U_{\lambda}\left(t_{0}\right)\right] e^{-\lambda\left(t-t_{0}\right)} \psi_{\lambda}(\vec{\rho}), \\
Q\left(\vec{\rho}, t \mid t^{\prime}\right)=\sum_{\lambda}\left[f_{\lambda}\left(t^{\prime}\right)-\dot{U}_{\lambda}\left(t^{\prime}\right)\right] e^{-\lambda\left(t-t^{\prime}\right)} \psi_{\lambda}(\vec{\rho}) .
\end{gathered}
$$

Let us summarize the solution derived above. It is given by (4.3), (4.4) and the two equations above, which, when joined, read

$T(\vec{\rho}, t)=U(\vec{\rho} \mid t)+\sum_{\lambda} \psi_{\lambda}(\vec{\rho}) e^{-\lambda t}\left\{\left[h_{\lambda}-U_{\lambda}\left(t_{0}\right)\right] e^{\lambda t_{0}}+\int_{t_{0}}^{t} d t^{\prime} e^{\lambda t^{\prime}}\left[f_{\lambda}\left(t^{\prime}\right)-\dot{U}_{\lambda}\left(t^{\prime}\right)\right]\right\}$.

In this,

$$
U_{\lambda}(t) \equiv \int_{\mathcal{A}} d A \psi_{\lambda}^{*}(\vec{\rho}) U(\vec{\rho} \mid t), \quad \dot{U}_{\lambda}(t)=\int_{\mathcal{A}} d A \psi_{\lambda}^{*}(\vec{\rho}) U_{t}(\vec{\rho} \mid t)=\frac{d U_{\lambda}}{d t},
$$


whereas $h_{\lambda}$ and $f_{\lambda}(t)$ are given in (2.8) and (3.7).

For the wave problem, the solution that would be obtained using the method above is

$$
\begin{gathered}
z(\vec{\rho}, t)=U(\vec{\rho} \mid t)+\sum_{\lambda} \psi_{\lambda}(\vec{\rho})\left\{\left[h_{\lambda}-U_{\lambda}\left(t_{0}\right)\right] C_{\lambda}\left(t-t_{0}\right)+\right. \\
\left.\left[v_{\lambda}-\dot{U}_{\lambda}\left(t_{0}\right)\right] S_{\lambda}\left(t-t_{0}\right)+\int_{t_{0}}^{t} d t^{\prime}\left[f_{\lambda}\left(t^{\prime}\right)-\ddot{U}_{\lambda}\left(t^{\prime}\right)\right] S_{\lambda}\left(t-t^{\prime}\right)\right\} .
\end{gathered}
$$

\section{Exemplification of the Methods}

\subsection{The exemplifying problem}

In this section, we apply the methods expounded in Sections 3 and 4 to solve the following heat problem in plane polar coordinates:

$$
T_{t}(\rho, \varphi, t)-\nabla^{2} T=f(\rho, \varphi, t), \quad T(1, \varphi, t)=g(\varphi, t), \quad T(\rho, \varphi, 0)=h(\rho, \varphi),
$$

with $t \geq 0,0 \leq \rho \leq 1, \varphi \in \mathbb{R}$, and under the condition of periodicity

$$
T(\rho, \varphi, t)=T(\rho, \varphi+2 \pi, t) .
$$

Its solution is the temperature in the disk of unit radius and centered at the origin of the $x y$ plane. This problem is a particular case of that in (2.1), with $\overline{\mathcal{A}}$ corresponding to the points of that disk, $\hat{\mathrm{B}}=1$ (Dirichlet boundary condition), and $t_{0}=0$.

First of all, we need to solve the two-dimensional eigenvalue problem specified by $(2.5)$, which here takes the form

$$
\psi_{\rho \rho}+\rho^{-1} \psi_{\rho}+\rho^{-2} \psi_{\varphi \varphi}+\lambda \psi(\rho, \varphi)=0, \quad \psi(1, \varphi)=0 .
$$

The substitution of the separation of variables approach $\psi=\mathcal{R}(\rho) \mathcal{F}(\varphi)$ gives

$$
\left(\frac{\mathcal{R}^{\prime \prime}+\rho^{-1} \mathcal{R}^{\prime}}{\mathcal{R}}+\lambda\right) \rho^{2}+\underbrace{\mathcal{F}^{\prime \prime} / \mathcal{F}}_{-\mu}=0 .
$$

We indicate above that the last term must be equal to the constant $-\mu$. This fact and the condition of periodicity (5.2) lead to the well known one-dimensional eigenvalue problem

$$
\mathcal{F}^{\prime \prime}+\mu \mathcal{F}(\varphi)=0, \quad \mathcal{F}(\varphi)=\mathcal{F}(\varphi+2 \pi),
$$

whose solutions (cf. Reference [1, Secs. 8.3 and 9.2]) are the eigenfunctions $\mathcal{F}_{m}(\varphi)=$ $e^{\mathrm{i} m \varphi}$, corresponding to the eigenvalues $\mu_{m}=m^{2}$, with $m=0, \pm 1, \pm 2, \cdots$.

With $\mu$ determined, we can solve the other 1D eigenvalue problem which follows from the radial ODE separated in (5.4) and the boundary condition in (5.3):

$$
\rho^{2} \mathcal{R}^{\prime \prime}{ }_{m}+\rho \mathcal{R}_{m}^{\prime}+\left(\lambda_{m} \rho^{2}-m^{2}\right) \mathcal{R}_{m}(\rho)=0, \quad \mathcal{R}_{m}(1)=0 .
$$


Actually, this is an eigenvalue problem for each value of $m$. The well known eigenvalues are $\lambda_{m n}=j_{m n}^{2}$ and the corresponding eigenfunctions are $\mathcal{R}_{m n}(\rho)=\mathrm{J}_{m}\left(j_{m n} \rho\right)$, where $j_{m n}$ is the $n$-th positive zero of the Bessel function $\mathrm{J}_{m}(n=1,2,3 \cdots)$ (cf. Reference [1, Sec. 9.7]).

Therefore, the eigenvalues of (5.3) and the corresponding eigenfunctions satisfying the normalization condition contained in (2.6) are

$$
\lambda_{m n}=j_{m n}^{2}, \quad \psi_{m n}(\rho, \varphi)=\frac{\mathrm{J}_{m}\left(j_{m n} \rho\right) e^{\mathrm{i} m \varphi}}{\sqrt{\pi} \mathrm{J}_{m+1}\left(j_{m n}\right)} .
$$

\subsection{Solution according to the first method}

The solution of (5.1) according to (3.9) is, using (5.5),

$T(\rho, \varphi, t)=\sum_{m=-\infty}^{\infty} \sum_{n=1}^{\infty} \psi_{m n}(\rho, \varphi) e^{-j_{m n}^{2} t}\left\{h_{m n}+\int_{0}^{t} d t^{\prime} e^{j_{m n}^{2} t^{\prime}}\left[f_{m n}\left(t^{\prime}\right)+B_{m n}\left(t^{\prime}\right)\right]\right\}$,

where $h_{m n}$ and $f_{m n}\left(t^{\prime}\right)$, according to (2.8) and (3.7), and using (5.5), are given by

$\left.\begin{array}{r}h_{m n} \\ f_{m n}\left(t^{\prime}\right)\end{array}\right\}=\int_{0}^{2 \pi} d \varphi \int_{0}^{1} d \rho \rho \psi_{m n}^{*}(\rho, \varphi)\left\{\begin{array}{l}h(\rho, \varphi) \\ f\left(\rho, \varphi, t^{\prime}\right)\end{array}=\int_{0}^{2 \pi} d \varphi \int_{0}^{1} d \rho \rho \frac{\mathrm{J}_{m}\left(j_{m n} \rho\right) e^{-\mathrm{i} m \varphi}}{\sqrt{\pi} \mathrm{J}_{m+1}\left(j_{m n}\right)}\left\{\begin{array}{l}h(\rho, \varphi) \\ f\left(\rho, \varphi, t^{\prime}\right)\end{array}\right.\right.$

In addition, according to $(3.5), B_{m n}\left(t^{\prime}\right)$ can be calculated as follows:

$$
B_{m n}\left(t^{\prime}\right)=\int_{0}^{2 \pi} d \varphi[\underbrace{\psi_{m n}^{*}(1, \varphi)}_{0} \frac{\partial T}{\partial \rho}(1, \varphi, t)-\underbrace{T(1, \varphi, t)}_{g(\varphi, t)} \frac{\partial \psi_{m n}^{*}}{\partial \rho}(1, \varphi)]
$$

which, using the boundary conditions in (5.3) and (5.1) as indicated above, and substituting $\psi_{m n}(\rho, \varphi)$ from $(5.5)$, becomes

$$
B_{m n}\left(t^{\prime}\right)=\frac{-\left.\frac{d J_{m}\left(j_{m n} \rho\right)}{d \rho}\right|_{\rho=1}}{\sqrt{\pi} J_{m+1}\left(j_{m n}\right)} \int_{0}^{2 \pi} d \varphi g(\varphi, t) e^{-\mathrm{i} m \varphi}=\frac{j_{m n}}{\sqrt{\pi}} \int_{0}^{2 \pi} d \varphi g(\varphi, t) e^{-\mathrm{i} m \varphi} .
$$

\subsection{Solution according to the second method}

The solution of the exemplifying problem (5.1) according to (4.7) is

$$
\begin{gathered}
T(\rho, \varphi, t)=U(\rho, \varphi \mid t)+\sum_{m=-\infty}^{\infty} \sum_{n=1}^{\infty} \psi_{m n}(\rho, \varphi) e^{-j_{m n}^{2} t}\left\{h_{m n}-U_{m n}(0)+\right. \\
\left.\int_{0}^{t} d t^{\prime} e^{j_{m n}^{2} t^{\prime}}\left[f_{m n}\left(t^{\prime}\right)-\dot{U}_{m n}\left(t^{\prime}\right)\right]\right\}
\end{gathered}
$$

In this, the $h_{m n}$ and $f_{m n}\left(t^{\prime}\right)$ are those in (5.7), and $U(\rho, \varphi \mid t), U_{m n}(0)$ and $\dot{U}_{m n}\left(t^{\prime}\right)$ are determined as follows: 
The function $U(\rho, \varphi \mid t)$ is obtained by solving the problem defined by (4.1), which here takes the form

$$
\nabla^{2} U(\rho, \varphi \mid t)=0, \quad U(1, \varphi \mid t)=g(\varphi, t) .
$$

The solution thereof is given by the well known Poisson's integral formula (Reference [2, Sec. 9.5])

$$
U(\rho, \varphi \mid t)=\frac{1}{2 \pi} \int_{0}^{2 \pi} d \phi \frac{\left(1-\rho^{2}\right)}{1-2 \rho \cos (\phi-\varphi)-\rho^{2}} g(\phi, t) .
$$

Therefore, from (4.8), and using (5.5), we see that

$$
\left.\begin{array}{l}
U_{m n}(0) \\
\dot{U}_{m n}\left(t^{\prime}\right)
\end{array}\right\}=\int_{0}^{2 \pi} d \varphi \int_{0}^{1} d \rho \rho \frac{\mathrm{J}_{m}\left(j_{m n} \rho\right) e^{-\mathrm{i} m \varphi}}{\sqrt{\pi} \mathrm{J}_{m+1}\left(j_{m n}\right)}\left\{\begin{array}{l}
U(\rho, \varphi \mid 0) \\
U_{t^{\prime}}\left(\rho, \varphi \mid t^{\prime}\right)
\end{array}\right.
$$

with, from (5.10),

$$
\left.\begin{array}{l}
U(\rho, \varphi \mid 0) \\
U_{t^{\prime}}\left(\rho, \varphi \mid t^{\prime}\right)
\end{array}\right\}=\frac{1}{2 \pi} \int_{0}^{2 \pi} d \phi \frac{\left(1-\rho^{2}\right)}{1-2 \rho \cos (\phi-\varphi)-\rho^{2}}\left\{\begin{array}{l}
g(\phi, 0) \\
g_{t^{\prime}}\left(\phi, t^{\prime}\right) .
\end{array}\right.
$$

\section{Proof of the Equality of Both Solutions}

It is natural to ask if the solution of the exemplifying problem given by (5.6) can be shown to be equal to that given by (5.9). In this section, we answer this affirmatively, but in a more general sense. Instead of restricting the proof to the specific problem of Section 5, we demonstrate below that both expressions (3.9) and (4.7) of the solution of the general heat problem given by (2.1) can be shown to be equal. [The same procedure described below is valid to demonstrate that the solution expressions (3.10) and (4.9), referring to the wave problem given by (2.2), can be show to be equal.]

By comparing (3.9) and (4.7), we see that, in each of these two expressions, the terms due to the source and the initial condition are equal. Therefore, we only need to show the equality of the terms due to the boundary condition, that is,

$\sum_{\lambda} \psi_{\lambda}(\vec{\rho}) e^{-\lambda t} \int_{t_{0}}^{t} d t^{\prime} e^{\lambda t^{\prime}} B_{\lambda}\left(t^{\prime}\right)=U(\vec{\rho} \mid t)-\sum_{\lambda} \psi_{\lambda}(\vec{\rho}) e^{-\lambda t}\left\{U_{\lambda}\left(t_{0}\right) e^{\lambda t_{0}}+\int_{t_{0}}^{t} d t^{\prime} e^{\lambda t^{\prime}} \dot{U}_{\lambda}\left(t^{\prime}\right)\right\}$.

Replacing the $U(\vec{\rho} \mid t)$ above by its generalized Fourier series $\sum_{\lambda} U_{\lambda}(t) \psi_{\lambda}(\vec{\rho})$ and equating the coefficients of $\psi_{\lambda}(\vec{\rho})$, we obtain

$$
\int_{t_{0}}^{t} d t^{\prime} e^{\lambda t^{\prime}} B_{\lambda}\left(t^{\prime}\right)=e^{\lambda t} U_{\lambda}(t)-e^{\lambda t_{0}} U_{\lambda}\left(t_{0}\right)-\int_{t_{0}}^{t} d t^{\prime} e^{\lambda t^{\prime}} \dot{U}_{\lambda}\left(t^{\prime}\right)
$$

Since the right-hand side of the equation above is equal to $\int_{t_{0}}^{t} d t^{\prime} e^{\lambda t^{\prime}} \lambda U_{\lambda}\left(t^{\prime}\right)$, which can be seen by performing an integration by parts, the equality of (3.9) and (4.7) will be demonstrated if we prove that

$$
U_{\lambda}(t)=(1 / \lambda) B_{\lambda}(t) .
$$


[In a similar manner, it can be shown that the equality of the wave problem solutions (3.10) and (4.9) follows from the same equation above.]

To prove (6.1), we make use of the well known integral representation

$$
U(\vec{\rho} \mid t)=\oint_{\partial \mathcal{A}} d s^{\prime}\left[\frac{\partial \Gamma}{\partial n^{\prime}}\left(\vec{\rho} \mid \vec{\rho}^{\prime}\right) U\left(\vec{\rho}^{\prime} \mid t\right)-\Gamma\left(\vec{\rho} \mid \vec{\rho}^{\prime}\right) \frac{\partial U}{\partial n^{\prime}}\left(\vec{\rho}^{\prime} \mid t\right)\right]
$$

(Reference [3, Sec.1.10]) of the solution of (4.1) in terms of Green's function $\Gamma\left(\vec{\rho} \mid \vec{\rho}^{\prime}\right)$. We also make use of the following well known formula (Reference [3, Sec. 3.12]), which furnishes $\Gamma\left(\vec{\rho} \mid \vec{\rho}^{\prime}\right)$ expanded in the eigenfunctions of the problem in (2.5):

$$
\Gamma\left(\vec{\rho} \mid \vec{\rho}^{\prime}\right)=-\sum_{\lambda^{\prime}} \lambda^{\prime-1} \psi_{\lambda^{\prime}}^{*}\left(\vec{\rho}^{\prime}\right) \psi_{\lambda^{\prime}}(\vec{\rho}) .
$$

Then, using (4.8) to calculate $U_{\lambda}(t)$, with $U(\vec{\rho} \mid t)$ given by (6.2) and (6.3), we obtain

$U_{\lambda}(t)=\int_{\mathcal{A}} d A \psi_{\lambda}^{*}(\vec{\rho}) \sum_{\lambda^{\prime}} \frac{1}{\lambda^{\prime}} \psi_{\lambda^{\prime}}(\vec{\rho}) \oint_{\partial \mathcal{A}} d s^{\prime}\left[\psi_{\lambda^{\prime}}^{*}\left(\vec{\rho}^{\prime}\right) \frac{\partial U}{\partial n^{\prime}}\left(\vec{\rho}^{\prime} \mid t\right)-\frac{\partial \psi_{\lambda^{\prime}}^{*}}{\partial n^{\prime}}\left(\vec{\rho}^{\prime}\right) U\left(\vec{\rho}^{\prime} \mid t\right)\right]$,

or, rearranging and using (2.6),

$$
U_{\lambda}(t)=\sum_{\lambda^{\prime}} \frac{1}{\lambda^{\prime}} S_{\lambda^{\prime}}(t) \underbrace{\int_{\mathcal{A}} d A \psi_{\lambda}^{*}(\vec{\rho}) \psi_{\lambda^{\prime}}(\vec{\rho})}_{\delta_{\lambda^{\prime} \lambda}}=\frac{1}{\lambda} S_{\lambda}(t),
$$

where (with the primes on $s^{\prime}$ and $\vec{\rho}^{\prime}$ dropped)

$$
S_{\lambda}(t) \equiv \oint_{\partial \mathcal{A}} d s\left[\psi_{\lambda}^{*}(\vec{\rho}) \frac{\partial U}{\partial n}(\vec{\rho} \mid t)-\frac{\partial \psi_{\lambda}^{*}}{\partial n}(\vec{\rho}) U(\vec{\rho} \mid t)\right] .
$$

Therefore, in view of (6.4), we prove (6.1) by proving that $S_{\lambda}(t)=B_{\lambda}(t)$. To show that this equation is true, let us subtract (3.5) from (6.5):

$$
S_{\lambda}(t)-B_{\lambda}(t)=\oint_{\partial \mathcal{A}} d s\left\{\psi_{\lambda}^{*}(\vec{\rho})\left[\frac{\partial U}{\partial n}(\vec{\rho} \mid t)-\frac{\partial T}{\partial n}(\vec{\rho}, t)\right]-\frac{\partial \psi_{\lambda}^{*}}{\partial n}(\vec{\rho})[U(\vec{\rho} \mid t)-T(\vec{\rho}, t)]\right\} .
$$

Notice that all we need to do to complete the proof is verify the vanishing of the right-hand side of the above equation. We do it by using the boundary conditions for $T, U$ and $\psi_{\lambda}$, given in (2.1), (2.5) and (4.1), respectively, taking (2.3) into account. The verification is immediate in the case of Dirichlet or Neumann conditions. For boundary conditions of the third type, since both $p(\vec{\rho})$ and $q(\vec{\rho})$ differ from zero, we first deduce that

$$
\hat{\mathrm{B}} \psi_{\lambda}^{*}(\vec{\rho})=p(\vec{\rho}) \psi_{\lambda}^{*}(\vec{\rho})+q(\vec{\rho}) \frac{\partial \psi_{\lambda}^{*}}{\partial n}(\vec{\rho})=0 \Rightarrow-\frac{\partial \psi_{\lambda}^{*}}{\partial n}(\vec{\rho})=\frac{p(\vec{\rho})}{q(\vec{\rho})} \psi_{\lambda}^{*}(\vec{\rho}) \quad[\vec{\rho} \in \partial \mathcal{A}]
$$

and then substitute it into the previous equation to obtain:

$$
S_{\lambda}(t)-B_{\lambda}(t)=\oint_{\partial \mathcal{A}} d s \frac{\psi_{\lambda}^{*}(\vec{\rho})}{q(\vec{\rho})}[\underbrace{\hat{\mathrm{B}} U(\vec{\rho} \mid t)}_{g(\vec{\rho}, t)}-\underbrace{\hat{\mathrm{B}} T(\vec{\rho}, t)}_{g(\vec{\rho}, t)}]=0 . \quad \text { QED. }
$$




\section{Final Comments}

With respect to the second method, the first one has the advantage that it does not presume the differentiability of the boundary data $g(\vec{\rho}, t)$. However, it has the disadvantage that the series solution which it produces converges less rapidly. In fact, the poorer rate of convergence of (3.9) and (3.10) in $\mathcal{A}$ is a consequence of the fact that each term of these series satisfies the homogeneous boundary condition in (2.5), what renders such series incapable of satisfying the non-homogeneous one in $(2.1)$, unless $g(\vec{\rho}, t) \equiv 0$.

Resumo. Dois métodos de se resolver uma classe ampla de problemas de calor e onda são descritos. Os problemas são bidimensionais e apresentam tanto nãohomogeneidade da equação diferencial e das condições iniciais e de fronteira quanto dependência temporal das fontes e dos dados iniciais e de fronteira. A igualdade das duas soluções correspondentes é estabelecida, e uma ilustração dos métodos é fornecida por meio de uma aplicação deles a um problema específico, resolvido em coordenadas polares.

\section{References}

[1] E. Butkov, "Mathematical Physics", Addison-Wesley Publishing Company, Reading, Massachusetts, 1973.

[2] F.B. Hildebrand, "Advanced Calculus for Applications", Second Edition, Prentice-Hall, Englewood Cliffs, New Jersey, 1976.

[3] J.D. Jackson, "Classical Electrodynamics", John Wiley \& Sons, Second Edition, New York, 1975.

[4] F. John, "Partial Differential Equations", Third Edition, Springer-Verlag, New York, 1978.

[5] K.R. Symon, "Mechanics", Third Edition, Addison-Wesley Publishing Company, Reading, Massachusetts, 1971.

[6] E.C. Zachmanoglou, D.W. Thoe, "Introduction to Partial Differential Equations with Applications", The Williams \& Wilkins Company, Baltimore, 1976. 